\title{
AC 2008-1442: SOME INTERESTING ENGINEERING PROBLEMS WITH OBJECTS OF SIMPLE GEOMETRY AND RELATIVELY COMPLEX MATHEMATICAL FORMULATION
}

\section{B. Sridhara, Middle Tennessee State University}

Dr. B. S. Sridhara is a professor in the Department of Engineering Technology and Industrial Studies at Middle Tennessee State University. He received his B.S.M.E. and M.S.M.E. degrees from Bangalore University and Indian Institute of Science, Bangalore, India. He received his M.S.M.E. and Ph. D. degrees from Stevens Institute of Technology, Hoboken, New Jersey, and Auburn University, Alabama. Dr. Sridhara has published several peer-reviewed articles in the areas of Acoustics, Vibration, finite element methods, and Engineering Education.

\section{Joseph Prince, Middle Tennessee State University}

Joseph W. Prince is a senior at Middle Tennessee State University majoring in Aerospace with minors in Mathematics and Engineering Technology. He is a member of the American Institute of Aeronautics and Astronautics (AIAA), Tripoli Rocketry Association, and served as Vice-President of The Space Elevator Team of MTSU. Joseph plans on continuing his education in graduate school with an academic and research emphasis on propulsions. 


\title{
Some Interesting Engineering Problems with Objects of Simple Geometry and Relatively Complex Mathematical Formulation
}

\begin{abstract}
:
There are several interesting engineering problems related to objects of simple geometry that involve relatively complex mathematics. We consider three different problems in the area of Mechanics. These problems are discussed in our undergraduate classes without getting into the mathematical details. In the ET 1840 - Engineering Fundamentals class we discuss the "brachistochrone" (path of shortest time) which is a cycloid. This is the trajectory of a point on a disk that rolls without slipping along a straight line. The equation involves the radial distance, and sine and cosine functions. This problem analyzed by Bernoulli is considered to be the foundation of the calculus of variation. We discuss the theory briefly in the class and let the students get the details from the course website if they desire to do so. Students are required to work in teams, and build a cycloidal- and straight-path model and test it. In the ET 4830 Vibration course, our students learn the theory of the Helmholtz resonator, build and test the device. The students learn to set up the equation of motion for the vibrating air mass in the resonator neck considering a small pressure difference. They derive an expression for the airspring stiffness under adiabatic conditions. They obtain an expression for the natural frequency of the resonator in terms of the resonator body and neck geometry. The students are required to design and build the resonator for a particular natural frequency using AutoDesk Inventor, and rapid prototyping and $\mathrm{CNC}$ machines. They test their resonator using a microphone and a PCbased software, and compare the theory and experiment.
\end{abstract}

In the vibration course we introduce the vibration of continuous systems and teach the basics of partial differential equations. Instead of using a standard problem from the textbook the author gives an overview of a panel absorber type muffler that can be used to reduce the noise propagated in a pipe or duct. The author has worked on this muffler for several years and published articles. A panel absorber consists of a rectangular panel (mass) backed by an air gap (spring). The student get an overview of how the equation of motion, which is a fourth-order partial differential equation, is derived involving the panel deflection, geometric and material properties of the device, and the time and space variables. The students get a chance to see a full-size panel absorber model and some insertion loss results. They also learn the effect of the cavity geometry, panel material and thickness, and boundary (mounting) conditions on the muffler performance. We are in the process of setting up a vibration lab where students can run standard experiments as well as design, build and test panel absorbers. 


\section{Introduction}

The Engineering Technology (ET) program at Middle Tennessee State University has three concentrations; computer, electromechanical and mechanical. The mechanical engineering technology (MET) concentration was started in 2004 fall and currently we have approximately one hundred majors. Our students are required to take several ET and MET courses including Engineering Fundamentals and Vibration. Our objective is to introduce hands-on projects in as many courses as possible so that the students better understand the theory and its applications to engineering systems and components. We feel there is a great need for such projects to alleviate the fear of mathematics in our students in courses such as Vibration. In this paper, we look at three different projects that are of simple geometry with relatively complex mathematical basis. In Engineering Fundamentals we discuss the mathematical formulation very briefly because it is a freshman course. In Vibration, which is a senior course, we discuss the mathematical formulation involving ordinary differential equations in some detail and briefly touch upon partial differential equations.

\section{The Cycloid Project}

This hands-on project is designed for the Engineering Fundamentals class. It is based on Johann Bernoulli's intriguing brachistochrone (the path of least time) problem proposed in June 1696. This well-known problem is to find the curve joining the two points, along which a particle falling from rest under the influence of gravity travels from the higher to the lower points (Figure 1) in the least time. ${ }^{1}$

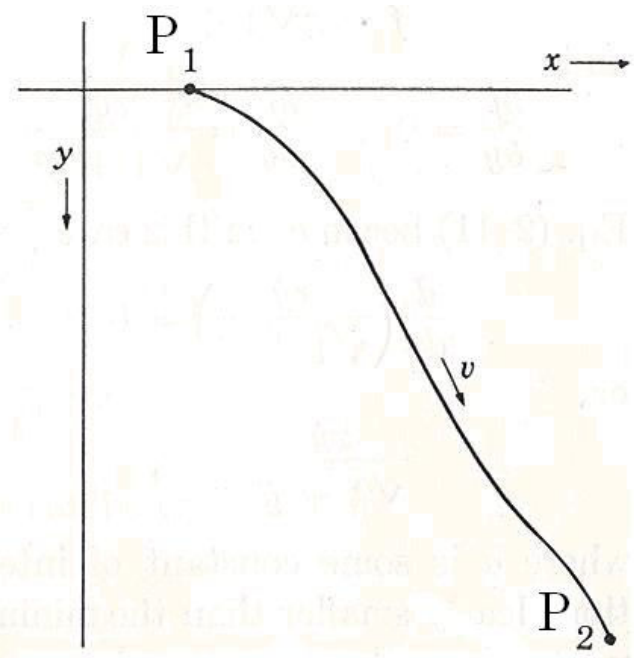

Figure 1. A curve along which a particle is falling under the action of gravity

The time required to travel from point $\mathrm{P}_{1}$ to point $\mathrm{P}_{2}$ is given by ${ }^{1,2}$

$$
t_{12}=\int_{F_{1}}^{F_{2}} \frac{d s}{v}
$$


Where $v$ is the particle's velocity and $d s$ is the arc length through which it is falling. Applying the law of conservation of energy we can write for the particle

$$
\frac{1}{2} m v^{2}=m g y
$$

Or

$$
v=\sqrt{2 g y}
$$

Substituting this for $v$ in the integrand of the first equation and following the steps given in References 1 and 2, we can show that

$$
\begin{aligned}
& x=1 / 2 k^{2}(q-\sin q) \\
& y=1 / 2 k^{2}(q-\cos q)
\end{aligned}
$$

Where $k^{2}$ is a constant and $x$ and $y$ describe a cycloid.

We discussed the above part very briefly in the class and showed the other mathematical details posted on the course site. Additional information on this problem and about the Bernoulli brothers, Newton and Leibniz ${ }^{3}$ was also posted on the course site.

Many of us know from engineering graphics that the construction of a cycloid is very simple. The Engineering Fundamentals students were assigned the cycloid project as a team project with a maximum of four students in each team. The author designed and built a 10-inchlong cycloidal- and straight-path model (Figures 2 and 3).

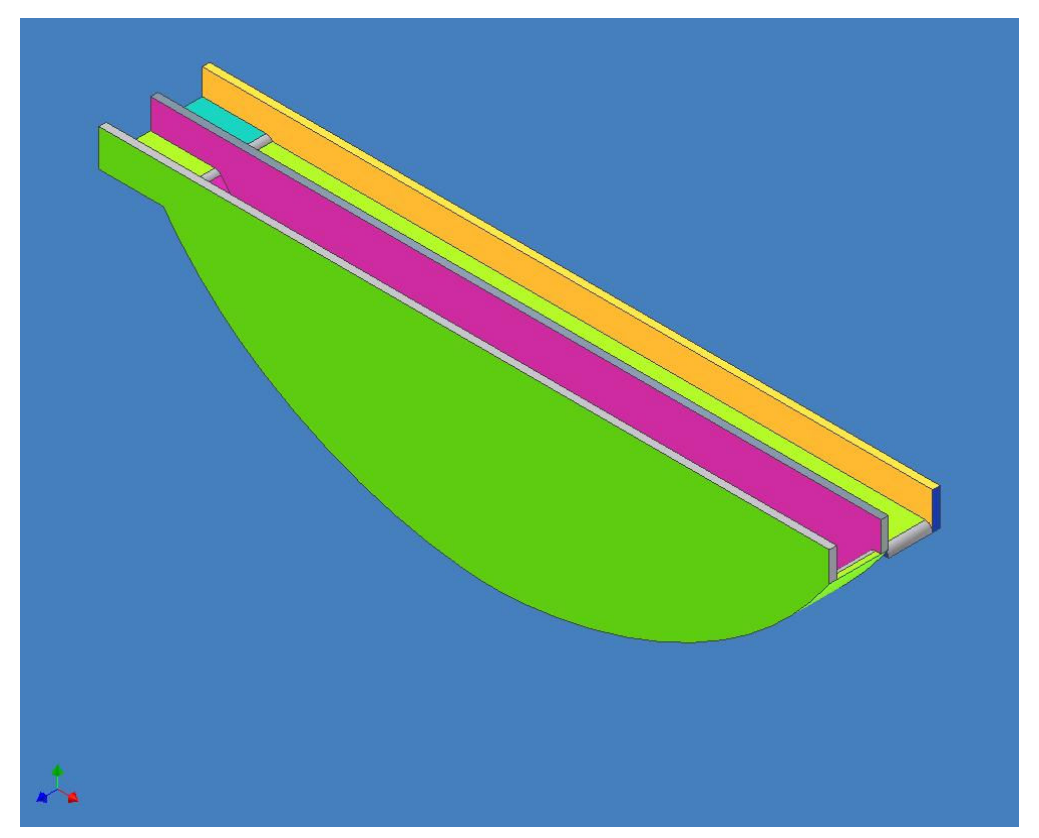

Figure 2. A cycloidal and straight path 3D model designed using AutoDesk Inventor. 


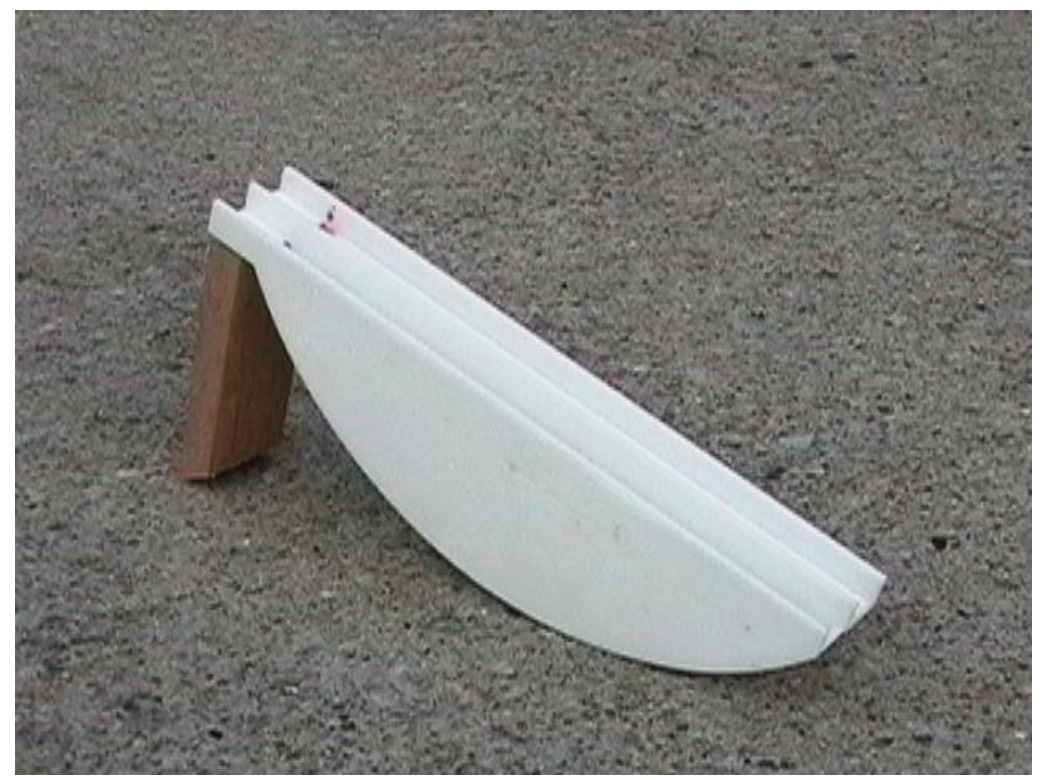

Figure 3. Plastic prototype of the cycloidal- and straight-path model built on the Stratasys rapid prototyping machine.

The demonstration consisted of rolling down two identical marbles simultaneously along the straight and curved paths. The students were unanimous in saying that the straight path would be the path of least time. But their opinion was changed after the demonstration and each student was allowed to roll down the marbles as demonstrated. Each student team was required to build an approximately two-foot long cycloid model similar to that shown in Figure 3 but had the choice of using cardboard, plastics, Styrofoam, sheet metal or plywood. The following step by step instructions were posted on the course site for constructing a cycloid graphically and building a model similar to that shown in Figure 3.

\section{Instructions for Graphically Constructing a Cycloid and building a Cycloidal- and Straight- Path Model Similar to that Shown in Figure 3:}

You can build the cycloidal- and straight-path unit out of materials such as cardboard, plywood, Styrofoam, or matallic and plastic sheets. You may use wood glue, Velcro, superglue, nails, etc. to assemble the parts together. You are encouraged to be creative and make your model aesthetically great.

1. Cut out a circular disk of at least 7 inches in diameter. Make a hole at 3 inches from the center of the circle so that a ball point or marker pen tip will clearly go through the hole.

2. Place a 3'x1' sheet material of your choice flat on a horizontal or vertical support and use adhesive tape or weights to prevent it from moving.

3. Place a 3'-long, $1 / 4$ "-thick straight edge such as a ruler aligning it with one long edge of the sheet material and fix it in position with adhesive tape or by other means.

4. Place the circular disk on the straight edge with the hole at the bottom. Insert a ball point or marker pen through the hole and rotate the circular disk one full revolution as shown in Figure 4. Make sure the disk will roll on the straight edge without slipping. 
5. If everything is done properly, you will see a cycloid generated by the moving pen as shown in Figure 4. Join the two end points of the cycloid with a straight line and your template is ready (Figure 5).

6. Make two pieces of the template out of the sheet material you intend to use and build the assembled cylcloidal and straight paths as shown in the Figure 3.

Additional information on the cycloid is posted on the course site.

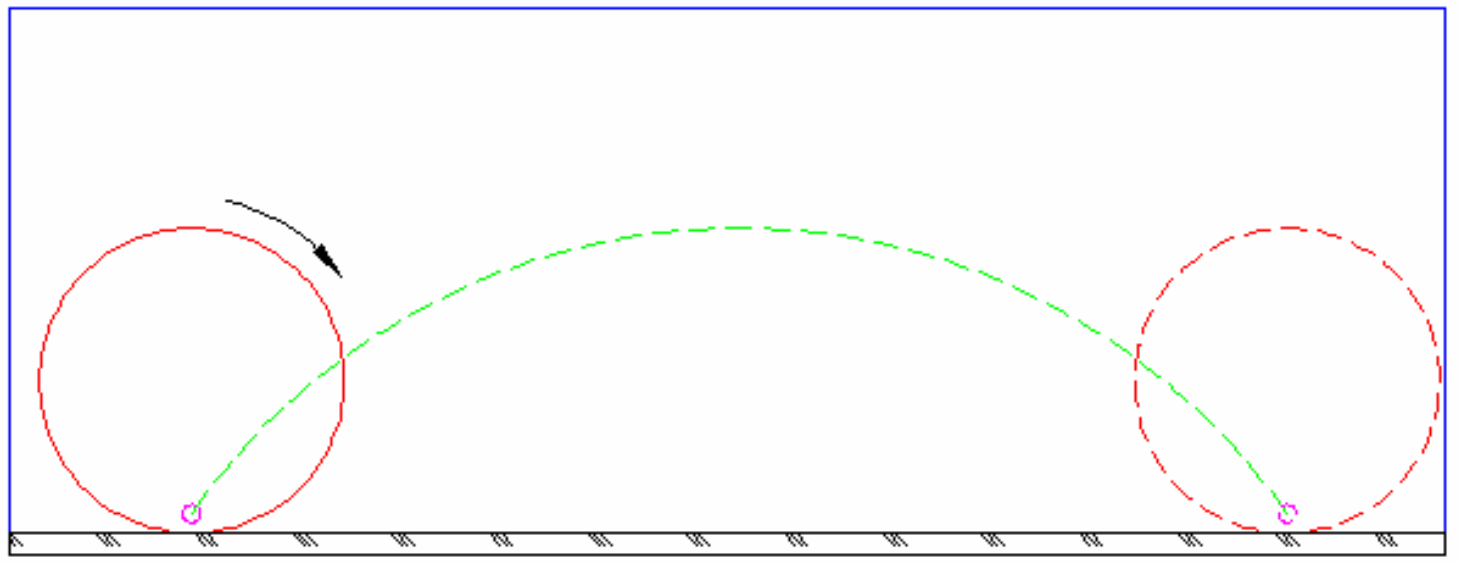

Figure 4. Generation of a cycloid

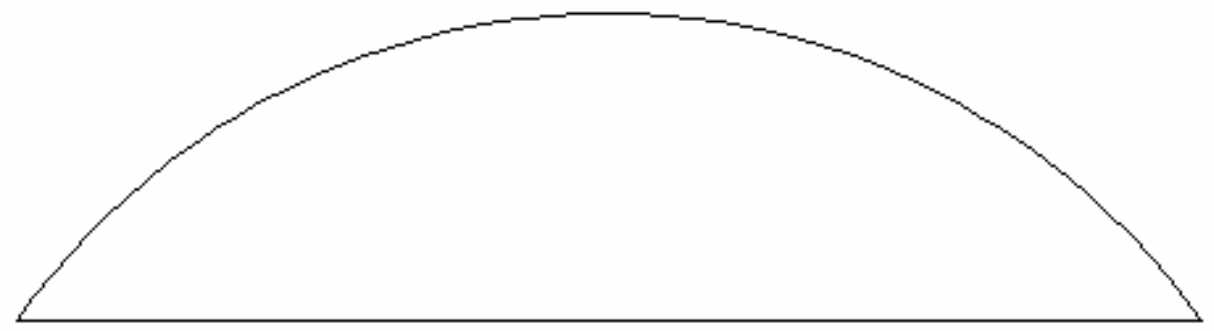

Figure 5. A template for the cycloid model

Student teams enjoyed displaying and repeatedly demonstrating their cycloid model on the day of their project presentation and Figure 6 shows a unit built by one student team. 


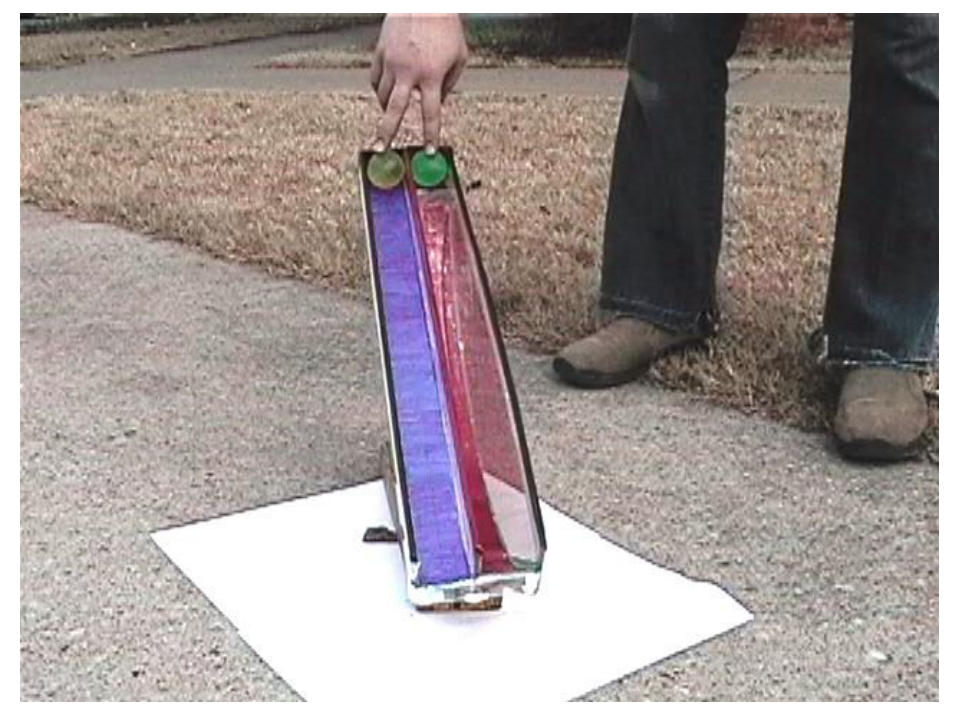

Figure 6. The cycloidal- and straight-path model built by one Engineering Fundamentals student team.

\section{Helmholtz Resonator}

So far we have taught Vibration in the fall semester of 2006 and 2007. We have introduced a hands-on project in this course while pursuing funding opportunities to establish a lab. We wanted the project to be a good learning experience in comparing the theory and experiment. There are several mechanical systems or devices that undergo oscillatory motion and can be modeled as spring-mass-damper systems. The damping in these could be seen as a separate entity as in the case of an automotive shock absorber or it could be an integral part of the system such as the internal/external friction in the case of a vibrating hack-saw blade (for example) mounted as a cantilever. It is not easy to find a pure spring-mass system because damping is always present in some form. However, there are some devices in which the damping is negligibly small and they can be approximated as pure spring-mass systems. The Helmholtz resonator is one such device. These are used in automotive mufflers in combination with simple expansion chambers. The theory of Helmholtz resonator is briefly discussed below.

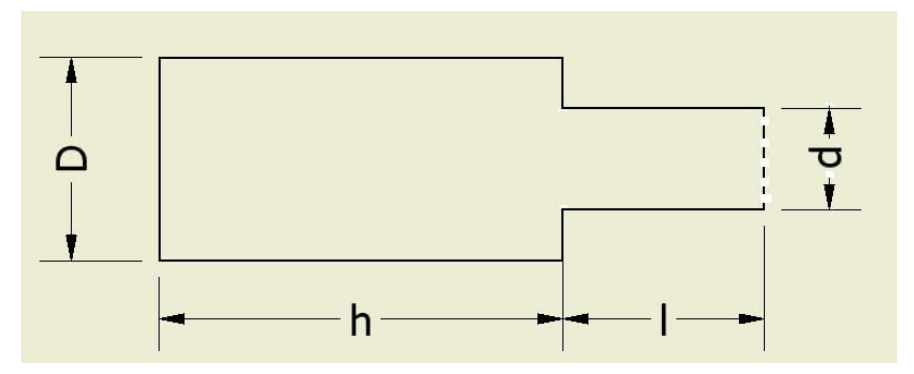

Figure 7. Schematic diagram of a Helmholtz Resonator: $\mathrm{d}=$ neck diameter; $\mathrm{D}=$ body diameter $1=$ neck length; $h=$ body length 
In the Helmholtz resonator (Figure 7) the volume of the air in the body functions as a spring and the air in the neck as the mass. The mass of air in the neck is given by ${ }^{4}$

$$
m=(l A)(\rho)
$$

Where $A=$ neck cross-sectional area,

$\rho=$ density of air.

The stiffness of the air spring is given by

$$
k=d p / d x
$$

Where $d p=$ the small pressure change that causes the oscillatory motion,

$d x=$ the elemental displacement of the air mass, $m$.

Considering adiabatic conditions during the oscillatory motion of the air mass we can write

$$
p V^{k}=\text { constant, }
$$

Where $p=$ air pressure in the resonator body,

$V=$ volume of the air in the resonator body,

$k=$ adiabatic index.

Following the steps given in Reference 4 we can write for the natural frequency of the above Helmholtz resonator as

$$
f_{0}=(c / 2 \pi)[A /(V l)]^{1 / 2},
$$

Where $c=$ speed of sound.

In the above expression $f_{0}$ is the undamped natural frequency in cycles per second (cps or $\mathrm{Hz}$ ) as the viscosity (damping property) of air is negligibly small.

The author simplified the expression for $f_{0}$ in terms of the resonator geometry shown in Figure 7 so that the students can work with different values of $(d / D),(h l)$ and a numerical constant for tuning the Helmholtz resonator to the desired natural frequency. Noting that the speed of sound in air is $c=343 \mathrm{~m} / \mathrm{s}$, the final expression for $f_{0}$ is given by

$$
f_{0}=5460(d / D)\left[1 /(h l)^{1 / 2}\right]
$$

Where $h$ and $l$ are measured in centimeters.

Designing, building and testing a Helmholtz resonator was a team project with a maximum of three members in a team. In 2006 fall the teams were asked to tune their resonator for a natural frequency of $300 \mathrm{~Hz}$. It was decided in the class to design the resonator using AutoDesk Inventor and build the unit directly from the 3D computer model using the 
department's rapid prototyping machine. This was to avoid human errors and obtain the best possible precision. However, we realized that the Stratsys prototyping machine could not build the resonator as one piece but in two pieces that had to be glued and taped together. Figure 8 shows three different ABS plastic resonators from 2006 fall.

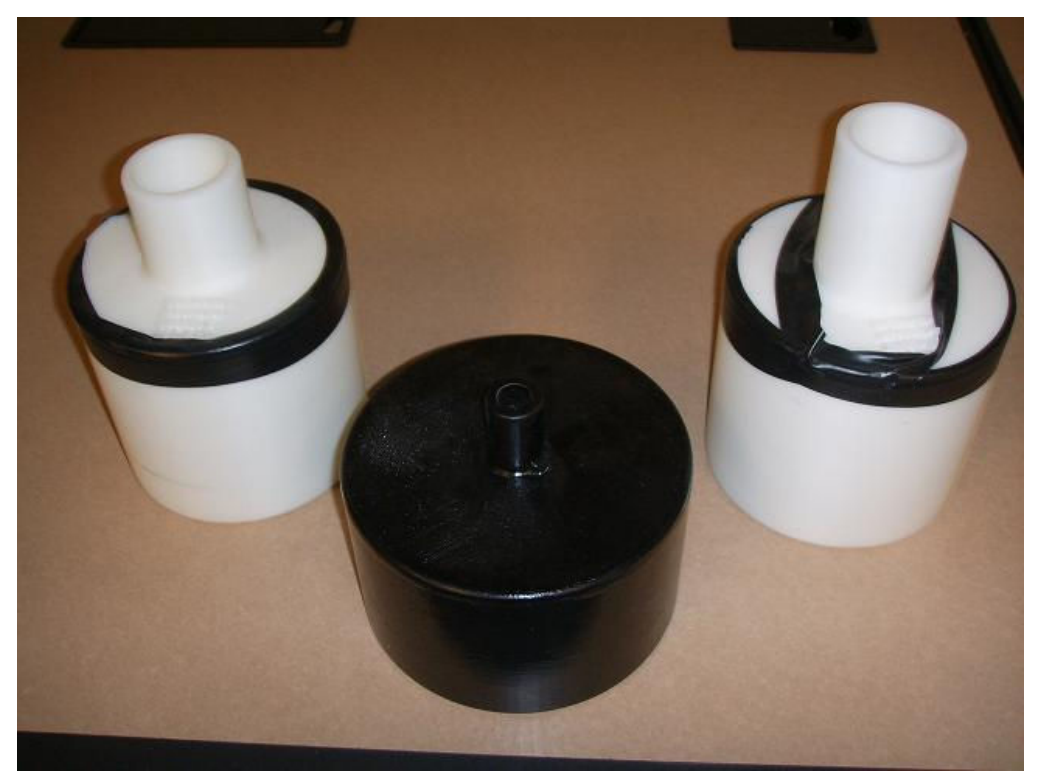

Figure 8. ABS plastic resonators built by some student teams in 2006 fall.

We used a laptop computer and a freeware (software) to test the resonators. The testing included pressing the resonator neck opening against our lower lip and quickly blowing once while the time domain data is recorded simultaneously. If everything was done correctly we should have seen a peak at $300 \mathrm{~Hz}$ which is the natural frequency of the resonator. The test results were very poor as there was no peak at any frequency in spite of several repetitions. One team member suggested that we pour some water into the resonator to decrease the air volume and thereby increase the natural frequency. The water started leaking out of the resonator and we realized that the porous ABS plastic walls were the reason for the resonators' poor performance.

In 2007 fall the vibration class decided to design the Helmholtz resonator using Inventor 2008 but build it using metals such as steel or aluminum. The author designed a resonator (Figure 9) using $(d / D)=0.5$ and $(h l)=8$ so that the natural frequency will be approximately $1000 \mathrm{~Hz}$. The bottom plate and the top cylindrical parts were press-fitted to avoid any air leakage. The student teams also tuned their resonator to an approximate natural frequency of $1000 \mathrm{~Hz}$ but used different $(d / D)$ and $(h l)$ values. One team even generated a series of different $d$, $D, h$ and $l$ values for the same $1000 \mathrm{~Hz}$ natural frequency. Steel and aluminum Helmholtz resonators built by student teams and the author are shown in Figure 10. 


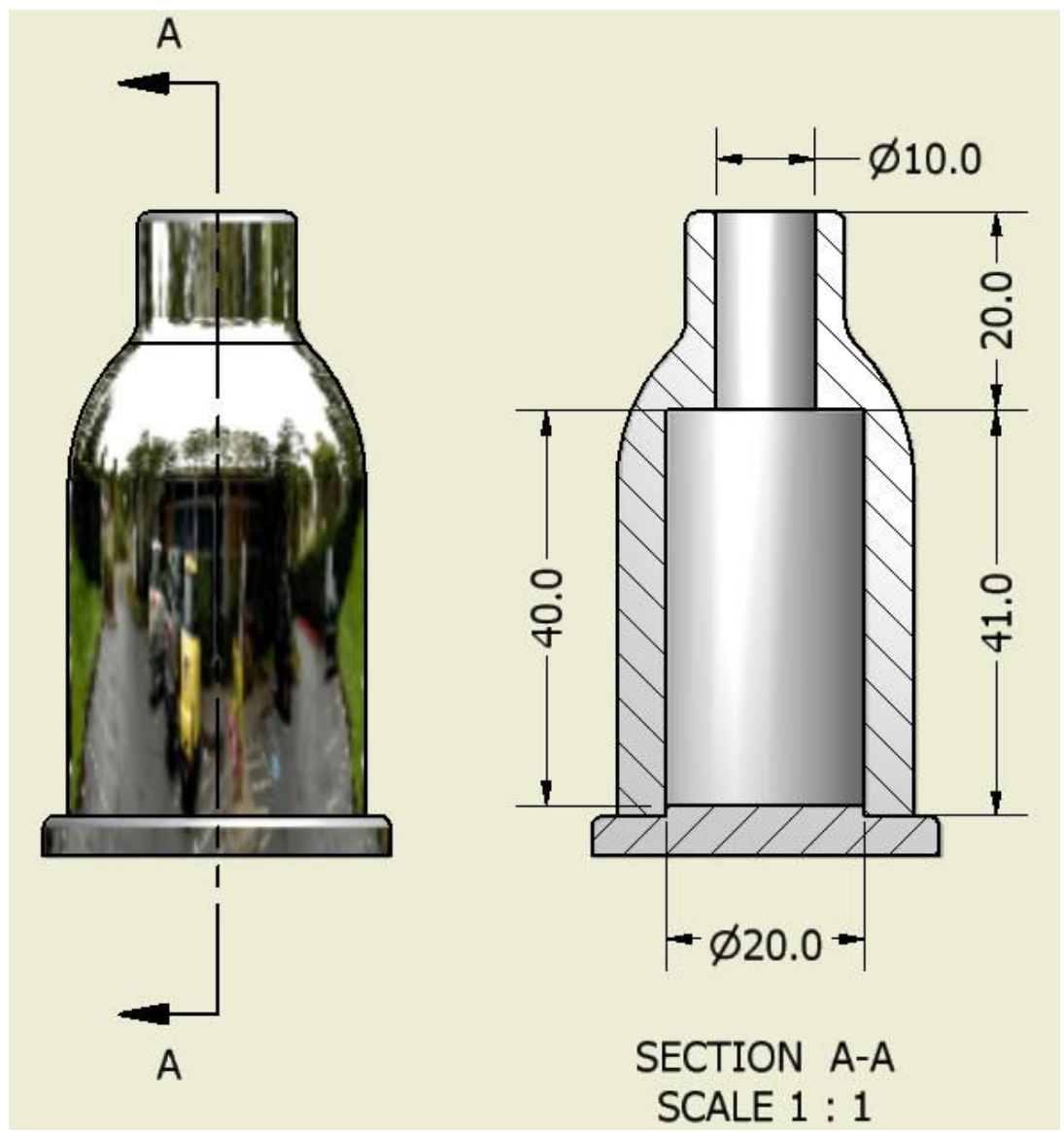

Figure 9. Full and sectional views of the 3D model of a Helmholtz resonator designed using Inventor 2008

The test set up in 2007 fall was the same as that used in 2006 fall. Some team members obtained the resonator response in the time domain by pressing the resonator neck opening against their lower lip and quickly blowing once. The others used the department's portable compressor for the same purpose while making sure the compressor was turned off after charging the air reservoir to the desired pressure. The time domain data indicated that all resonator responses showed a sinusoidal curve. The coauthor carried out further analysis of this data and obtained the frequency response for a typical resonator. The freeware and two other software were used to obtain the resonator response in the frequency domain. 


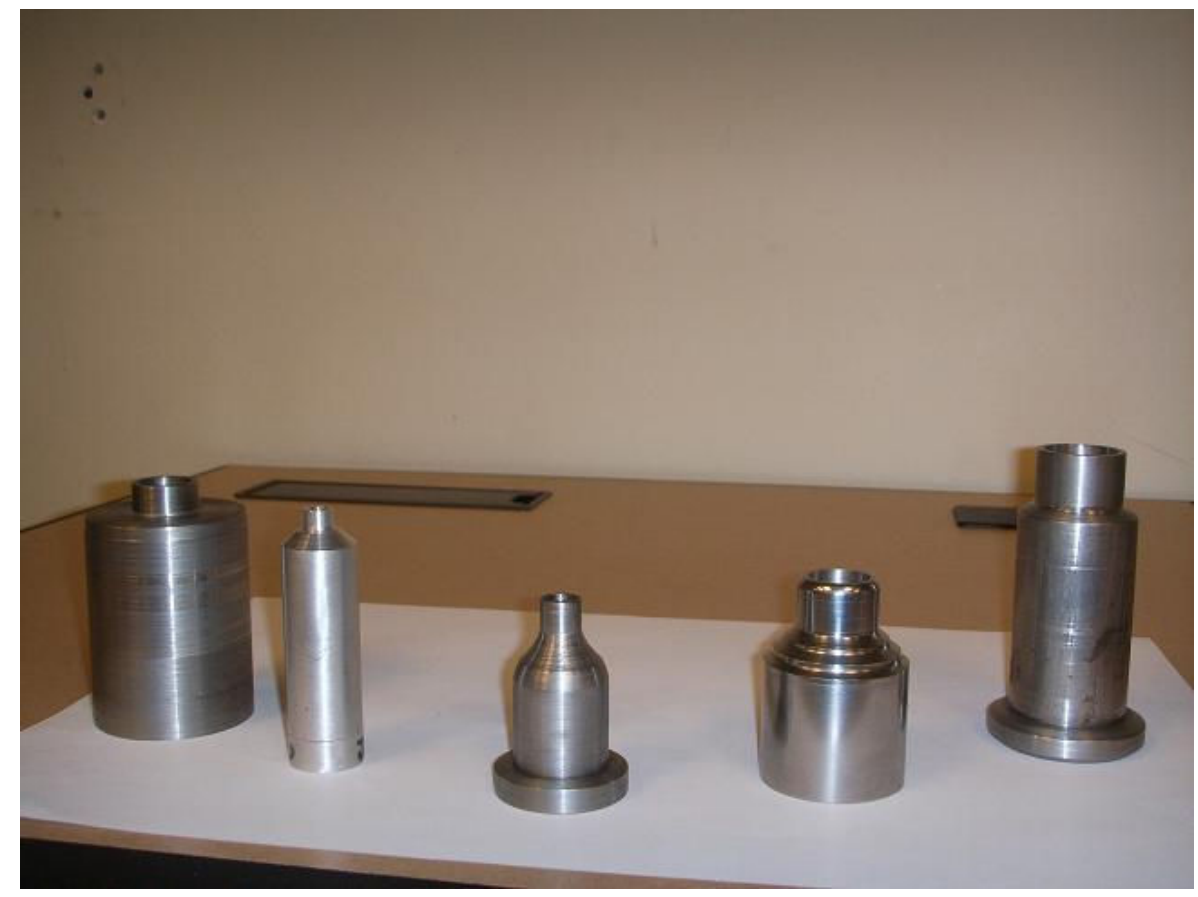

Figure 10. Steel and aluminum Helmholtz resonators built by student teams and the author

The freeware was found to be inadequate for the frequency analysis because when the time interval, for example, was increased by $2.844 \mathrm{~ms}$ the frequency suddenly decreased from $943 \mathrm{~Hz}$ to $732 \mathrm{~Hz}$ which is unrealistic. We used the trial version of another software to obtain the resonator frequency response. This software performed the fast Fourier transform (FFT) on the selected short time interval. However, it showed a peak at exactly $678.5 \mathrm{~Hz}$ for all resonators which was not right as each resonator was designed to respond at a natural frequency of approximately $1000 \mathrm{~Hz}$. Besides, each team used slightly different resonator dimensions and built them at different facilities. We also noticed that an increase of $3.525 \mathrm{~ms}$ in the time interval resulted in a frequency increase from $678.5 \mathrm{~Hz}$ to $873 \mathrm{~Hz}$ for one resonator, but the others still remained a constant $678.5 \mathrm{~Hz}$.

We tried a software that was relatively more expensive and it gave us more stable and better results. The Hanning window was selected because it was found to give the best frequency resolution. In this case when the time interval was increased by $3 \mathrm{~ms}$ the frequency only changed from $778 \mathrm{~Hz}$ to $777 \mathrm{~Hz}$ providing a consistency and confidence in the results which we did not find with the other software. Two examples of time domain data and frequency response are shown in Figures 11 and 12. A peak around $800 \mathrm{~Hz}$ (instead $1000 \mathrm{~Hz}$ ) is seen in both cases and we believe air damping and inner surface finish could have contributed to this shift in the natural frequency. 


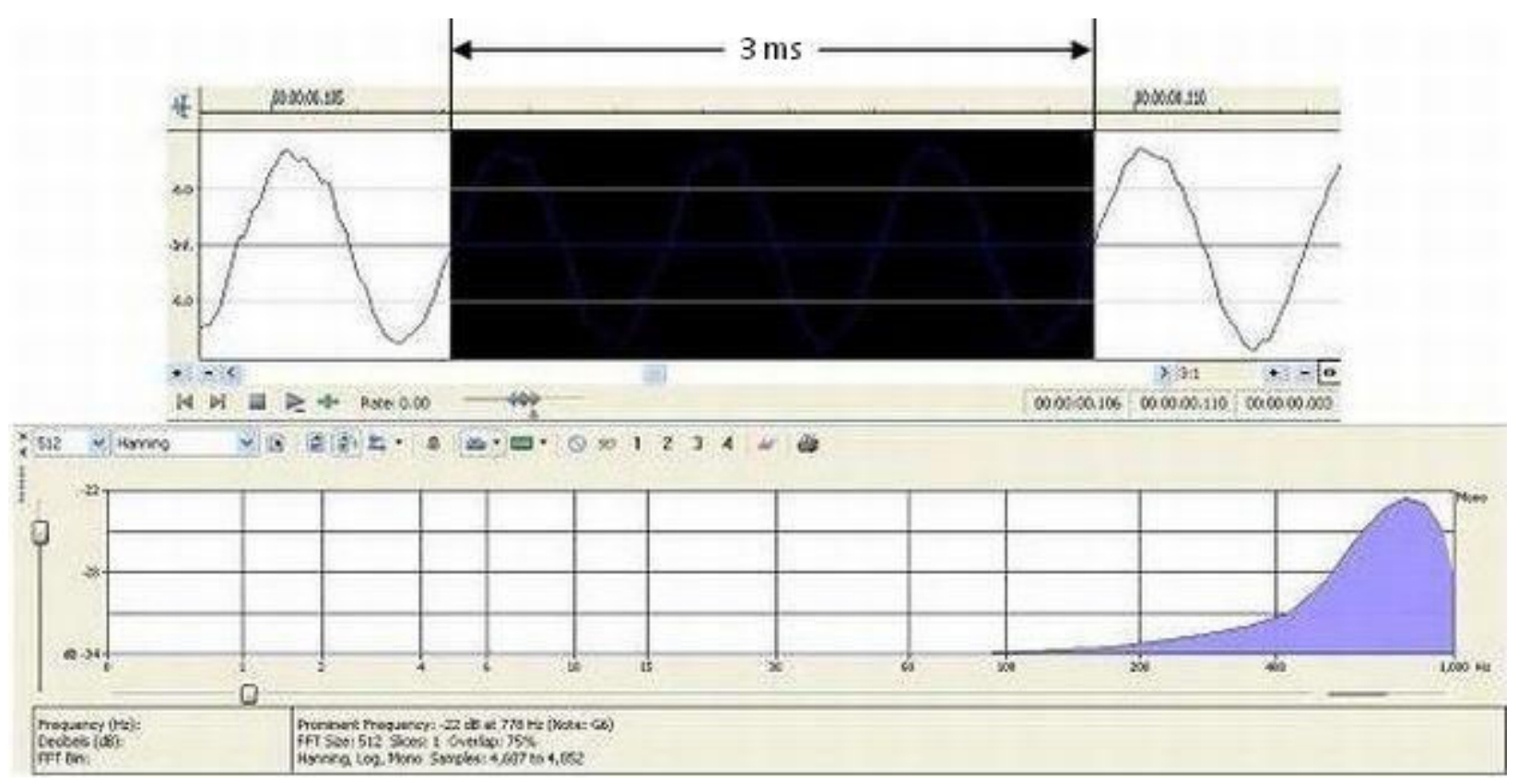

Figure 11. Time domain data and frequency response of a typical Helmholtz resonator built by students in 2007 fall.

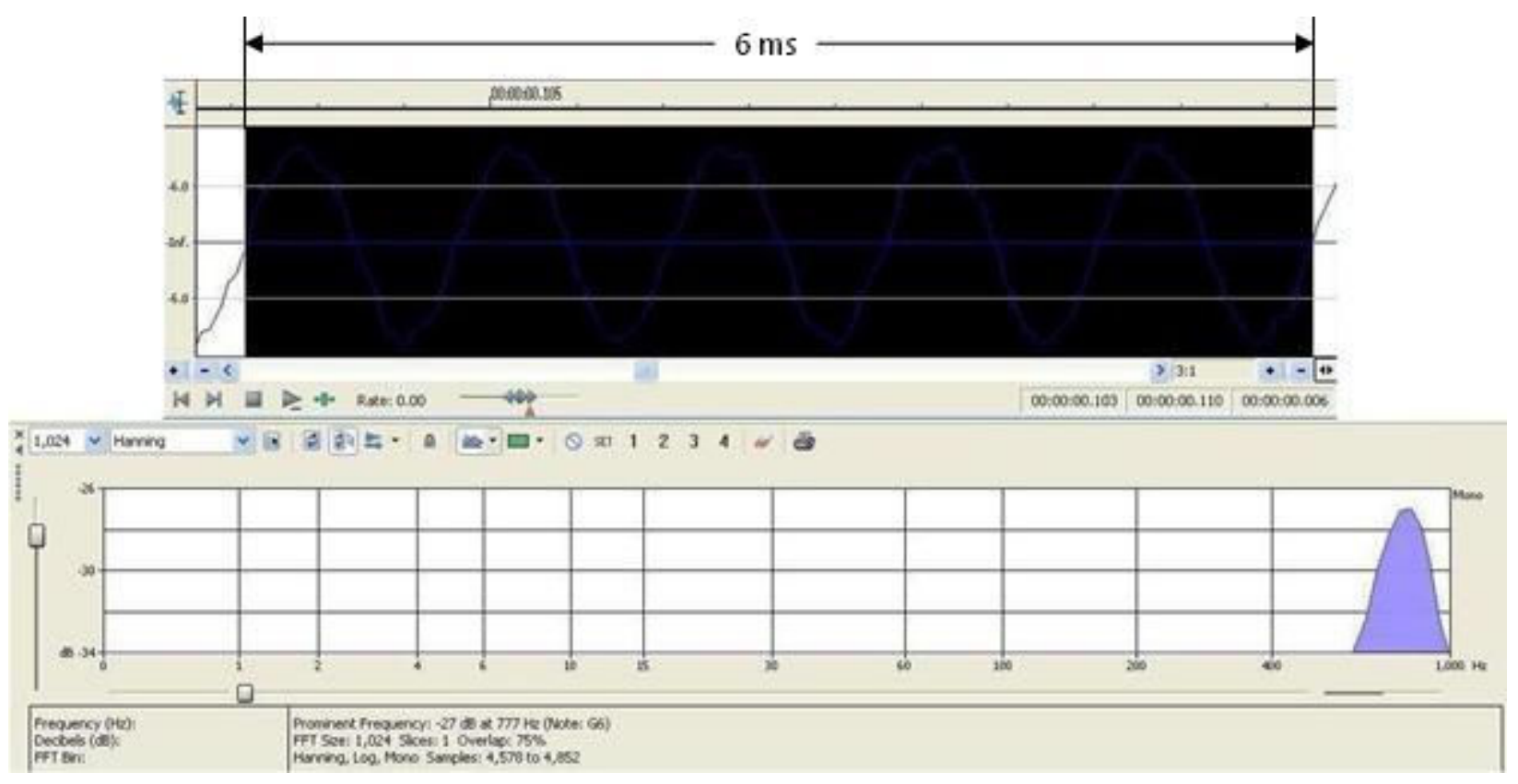

Figure 11. Time domain data and frequency response of a typical Helmholtz resonator built by students in 2007 fall. 


\section{Panel Absorbers}

A panel absorber used in a pipe or duct system is a reactive type muffler. Insertion loss is a measure of muffler performance. A panel absorber consists of a rectangular panel backed by an air gap (Fig. 12). It is a spring-mass system with the air acting as a spring and the panel as a mass. The panel absorber mounted in a pipe vibrates when there is sound propagation, reflects a part of the sound towards the source and thereby reduces the noise level at the pipe exit. Unlike the conventional mufflers, it is simple in construction, compact and there are no holes or open cavities. Therefore, panel absorbers do not get clogged and can also be used to reduce the noise propagated in pipes and ducts that are used to transport solid particles.

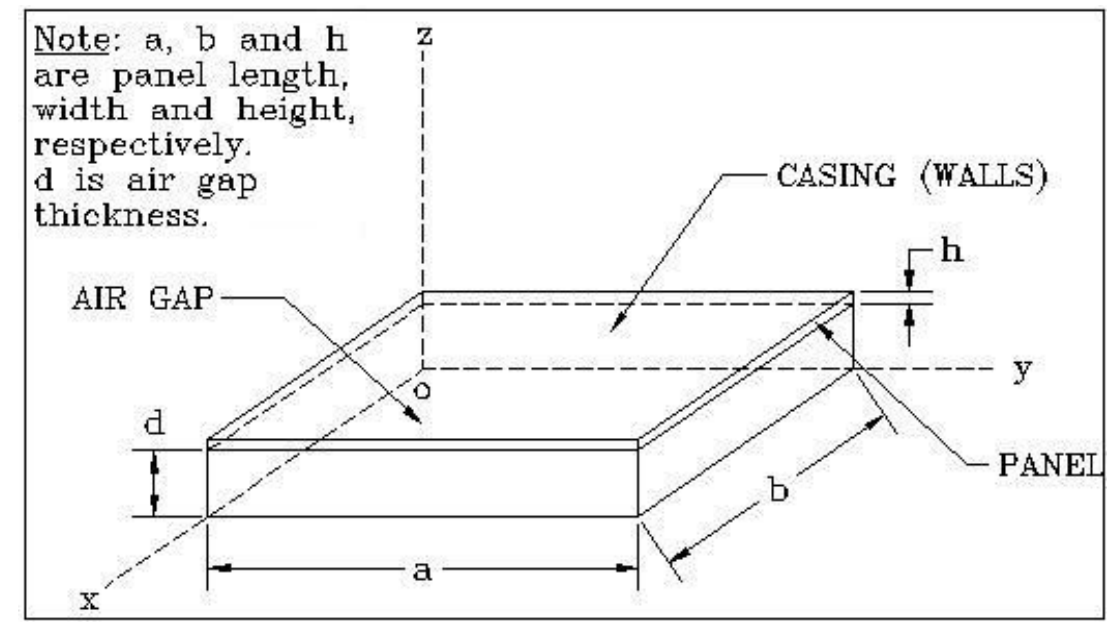

Figure 12. Schematic diagram of a typical panel absorber

When a panel absorber is modeled as a Helmholtz resonator we obtain an expression for its natural frequency as ${ }^{5}$

$$
f_{0}=(c / 2 \pi)\left[\rho_{\mathrm{A}} /(m d)\right]^{1 / 2},
$$

Where $c=$ speed of sound in air,

$\rho_{\mathrm{A}}=$ density of air,

$m=$ panel mass per unit area,

$d=$ air gap thickness.

Our Vibration students could relate to this approach as they have designed, built and tested a Helmholtz resonator. The author has designed, built and tested several aluminum panel absorbers made out of 10X8X 0.015 inch and 15X8X0.015 inch rectangular panels ${ }^{6}$ with several edge support or boundary conditions. Results in Reference 6 show that they respond at several frequencies and not just at the Helmholtz resonator natural frequency. This is because of the boundary effects that excite higher modes of vibration. The author used the energy method to develop the equation of motion of a panel absorber (which is a continuous system) using the distributed spring-mass system approach. ${ }^{7}$ In Reference 7 , panel absorber natural frequencies 
have been calculated by selecting several nodes along the length and width of the panel. Without going into the mathematical details we will write the panel absorber equation of motion as

$$
\rho_{\mathrm{p}} h \frac{\partial^{2} w}{\partial t^{2}}+\frac{E h^{3}}{12\left(1-v^{2}\right)}\left[\frac{\partial^{4} w}{\partial x^{4}}+2 \frac{\partial^{4} w}{\partial x^{2} \partial y^{2}}+\frac{\partial^{4} w}{\partial y^{4}}\right]+\frac{\rho_{\mathrm{a}} c^{2}}{d} w=0,
$$

Where $\rho_{P}=$ panel material density,

$\rho_{\mathrm{a}}=$ air density,

$h=$ panel thickness,

$d=$ air gap thickness,

$w=$ panel deflection perpendicular the panel,

$E=$ Young's modulus of panel material,

$v=$ Poisson's ratio.

The above is a fourth-order partial differential equation in time $t$, and space variables $x$ and $y$. In the class we very briefly discussed partial differential equations and showed how the vibration of a panel absorber of the simple geometry as shown in Figure 12 is governed by a complex equation of motion. As experimental work on aluminum panel absorbers were done several years ago at a different university a panel absorber model made out of plywood and cardboard (Figure 13) was used in the classroom to describe the working of a panel absorber.

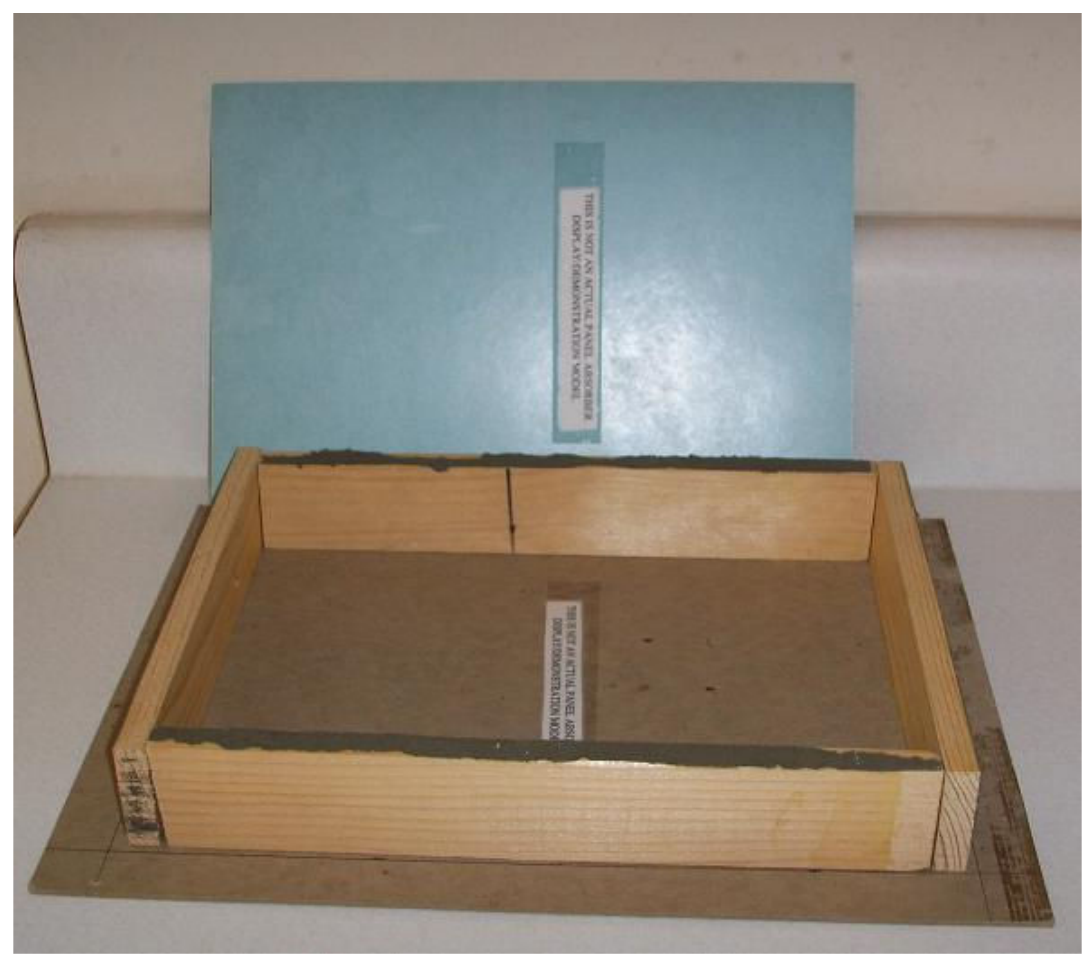

Figure 13. A panel absorber model made out of plywood and cardboard 


\section{Conclusions}

We believe an instructor needs to teach not only from the textbook but also from his/her industrial and research experience. Appropriately the author used his panel absorber research project to introduce partial differential equations in ET 4830 - Vibration. The students appeared to be more comfortable with the mathematics part when they saw the physical model consisting of the panel (mass) and the air gap (stiffness). They could also relate the physical aspects of the device to the mathematical terms. We intend to involve ET undergraduates participating in the STEPmt program (http://www.mtsu.edu/ stepmt/) in the further studies of the panel absorber project. The author has adopted a similar combination of theory and hands-on projects approach in ET 3830 - Statics (The Beam Project to show the effect of the section modulus on the beam deflection) and ET 3840 - Dynamics (The Egg Drop Contest to show the difference between kinematics and kinetics of a particle). He plans to develop such projects in courses such as Design of Machine Elements and Fluid Power.

Our MET concentration was started in 2004 fall it has been accredited by ABET for six years starting form 2007 fall. We have a continuous improvement plan for MET and the major field test (MFT) is one of the most significant tools used in the plan. There are one hundred questions in the MFT and we follow the norm of changing or modifying only five percent of the questions in a year. We have identified all one hundred questions of the MFT with the thirteen concentration outcomes which are formulated based on the ABET's a-k criteria. Currently there are no questions from Vibration in the major field test as we started teaching this senior-level course formally from 2006 fall. We plan to include questions from Vibration in the MFT for the 2008-09 academic year.

It is our experience that the hands-on projects help our students understand the theory better. This is very true in mathematics-intensive courses such as Vibration where students deal with differential equations and solutions. In the Engineering Fundamentals class the students can qualitatively see the difference between theory and practice in engineering. In summary, these projects have been a good learning experience for the students as well as the instructor.

\section{Bibliography:}

1. Herbert Goldstein, "Classical Mechanics,” Addison-Wesley Publishing Company, Inc., 1959.

2. URL: http://mathworld.wolfram.com/BrachistochroneProblem.html

3. URL: http://scienceworld.wolfram.com/biography/BernoulliJohann.html

4. Van Santen, G.W., Introduction to a Study of Mechanical Vibration," Philips Technical Library, 1958.

5. B. S. Sridhara, "Preliminary studies on the effectiveness of aluminum panel absorbers in a pipe system," Proceedings of Noise-Con 85, Columbus, Ohio, June 1985.

6. B. S. Sridhara, "Performance evaluation of small panel absorber type mufflers using the distributed springmass system approach," final report on summer research submitted to the dean of graduate studies, September 1997. 